\section{Check for updates}

Cite this: J. Mater. Chem. A, 2017, 5, 8690

\title{
Design of electric field controlled molecular gates mounted on metal-organic frameworks $\uparrow$
}

\begin{abstract}
Benjamin Tam and Ozgur Yazaydin (D) *
In this study we propose and computationally demonstrate the concept of electric field controlled molecular gates mounted on the open-metal coordination sites in metal-organic framework (MOF) materials. The MOFmolecular gate complex functions by opening and closing under the effect of an electric field. Our design involves Mg-MOF-74, a MOF with hexagonal channels with open-metal coordination sites at each corner, and a multifunctional gate molecule with permanent dipole which anchors itself on the host MOF material and responds to changes in the direction of an electric field by rotating around its backbone which acts as an axle. By carrying out density functional theory (DFT) calculations and molecular dynamics (MD) simulations we show that the MOF-molecular gate complex can be switched between two stable configurations, open and closed, by turning on and off an external electric field. We further show that the molecular gate can be controlled to block or allow the diffusion of methane molecules through the channels of the MOF like a nanoscale butterfly valve. Electric field controlled molecular gates mounted on MOFs can pave the way for new molecular machines and nanodevices which can store, deliver or select molecules on demand and with atomic precision.
\end{abstract}

Received 4th January 2017

Accepted 16th April 2017

DOI: $10.1039 / \mathrm{c} 7 \mathrm{ta00101k}$

rsc.li/materials-a

and shape and chemical functionality of MOFs. Thanks to such

\section{Introduction}

Molecular machines are supramolecular structures designed to transform external stimuli to coordinated collective mechanical movements which mimic those occurring at the macroscopic level. ${ }^{\mathbf{1} 2}$ Examples of molecular machines are rotors, ${ }^{3}$ switches, ${ }^{4-6}$ gates, ${ }^{7,8}$ elevators, ${ }^{\mathbf{9}, 10}$ motors ${ }^{\mathbf{6}, 11-13}$ and tweezers, ${ }^{\mathbf{1 4}}$ most of which were inspired by nature. ${ }^{15,16}$ More sophisticated designs include molecular machines which can move objects 10000 times greater than their mass ${ }^{17}$ and a molecular car. ${ }^{18,19}$ In the mid-1990s, Bedard et al. ${ }^{20}$ was the first to suggest the concept of electric field controlled molecular doorways by employing dipolar molecules. Later Zheng et $a .^{21}$ synthesized a molecular rotor and characterized its response to an electric field by scanning tunnelling microscopy and barrier height imaging. Horinek et al., ${ }^{22} \mathrm{Hsu}$ et al. ${ }^{23}$ and Seldenthuis et al. ${ }^{24}$ reported molecular simulations of similar unidirectional rotational control of supramolecular machines by an electric field.

Another area developing rapidly, like molecular machines, and have harnessed great interest recently, is metal-organic frameworks (MOFs). ${ }^{25-29}$ MOFs are inorganic-organic hybrid porous crystalline materials formed by the self-assembly of metal ions and organic ligands via coordination bonds. In principal, with a judicious choice of the inorganic and the organic building blocks it is possible to fine tune the pore size

Department of Chemical Engineering, University College London, Torrington Place, London, WC1E 7JE, UK. E-mail: ozgur.yazaydin@ucl.ac.uk

$\dagger$ Electronic supplementary information (ESI) available. See DOI: 10.1039/c7ta00101k versatility MOFs have been investigated for a wide range of applications, such as separations, ${ }^{30}$ gas storage, ${ }^{31,32}$ drug delivery, ${ }^{28,33,34}$ sensors, ${ }^{28,35,36}$ luminescence ${ }^{35,37}$ and catalyst. ${ }^{38-40}$ Structural and chemical properties of MOFs can also be tuned by post-synthesis modification. ${ }^{\mathbf{4 1 - 4 3}}$ This is usually achieved by ligand functionalization or grafting molecules on open-metal coordination sites. The latter forms the basis of our study. By grafting ligands on open-metal coordination sites, majority of previous studies aimed at tuning the pore size and chemistry of MOFs for various separation and catalyst applications. ${ }^{43-50}$ More recently Stoddart and colleagues demonstrated the idea of positioning redox-active molecular switches within MOFs by grafting them on the open-metal coordination sites. ${ }^{51,52}$

Propelled by the advancement in both synthetic molecular machines and MOFs, here we propose to mount molecular gates on the open-metal sites of MOFs and control their opening and closing by utilizing changes in an electric field. ${ }^{\mathbf{2 0 , 2 1 , 2 3 , 2 4}}$ The molecular gate consists of three basic features; anchors, a permanent dipole and an axle. The strong attraction provided by the open-metal coordination sites in MOFs provide anchoring points for the gate molecule and the permanent dipole allows the gate molecule to be influenced by an electric field and rotate around an axle to block or allow the diffusion of molecules in a channel. In our MOF-molecular gate design we first identified a MOF suitable for hosting a gate molecule. M-

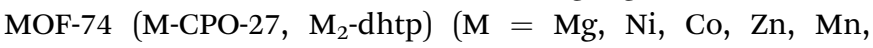
$\mathrm{Fe})^{54-56}$ materials have been studied for the adsorption of different species, including light gases and hydrocarbons. ${ }^{55,57-66}$ M-MOF-74 is composed of 2,5-dihydroxyterephthalate ligands 
a)

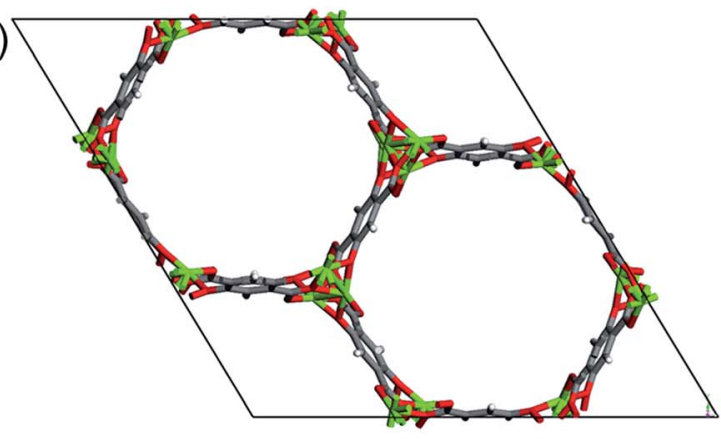

c)

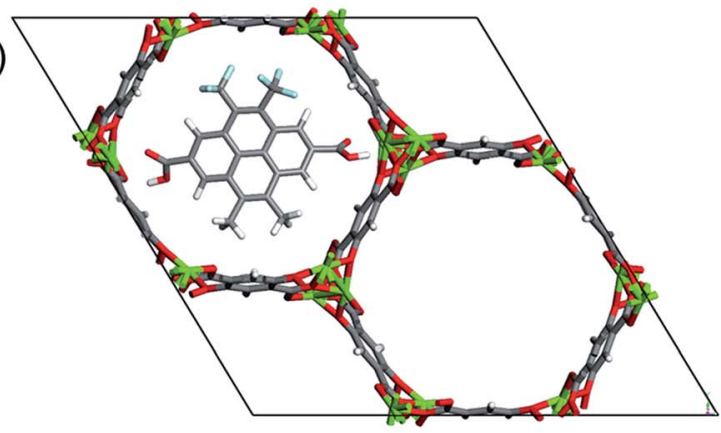

b)

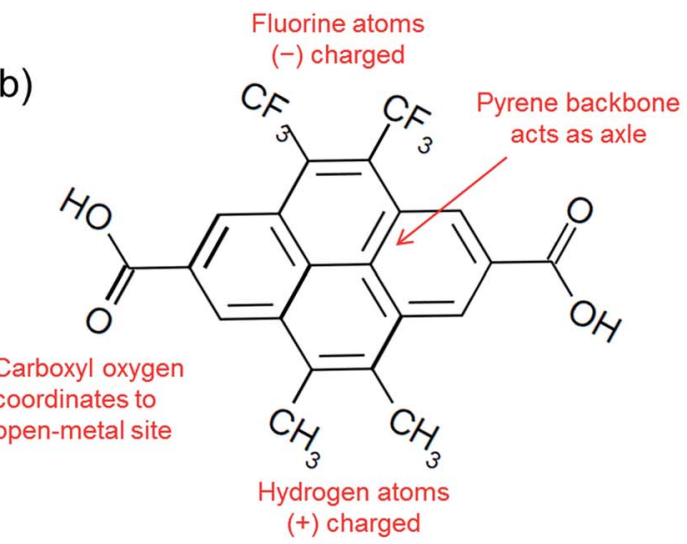

d)

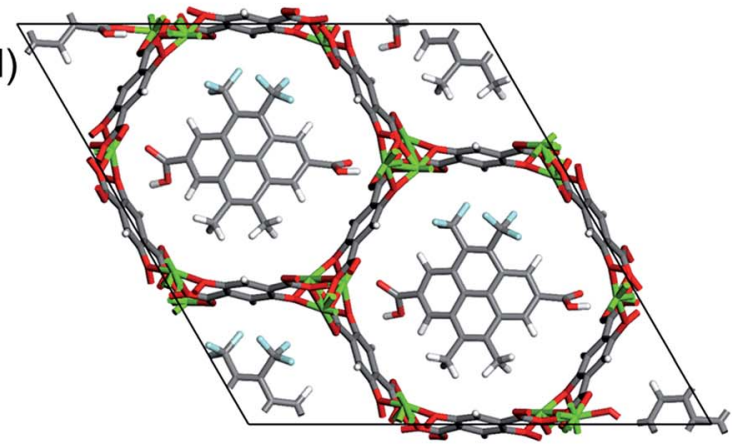

Fig. 1 (a) Mg-MOF-74 unit cell, (b) chemical structure of the gate molecule, 4,5-dimethyl-9,10-bis(trifloromethyl)pyrene-2,7-dicarboxylic acid, $^{53}$ (c) a molecular gate placed in one of the Mg-MOF-74 channels, and (d) MOF-molecular gate complex where all three channels of MgMOF-74 were occupied by the gate molecules. Carbon, oxygen, magnesium, fluorine and hydrogen are represented with grey, red, green, cyan and white, respectively.

and one of the named metal ions, forming a structure with hexagonal one-dimensional channels which are about $12 \AA$ in size and bear a high density of open-metal coordination sites. The open-metal sites facing one another in M-MOF-74 provide anchoring points in order to mount the gate molecules and its one-dimensional channels are ideal for the demonstration of the gating effect; i.e. closing or opening the pore. Of particular interest to us is Mg-MOF-74 (Fig. 1a) due to the strong binding energies reported for various adsorbates. ${ }^{67,68}$

For the gate molecule, we considered 4,5-dimethyl-9,10bis(trifloromethyl)pyrene-2,7-dicarboxylic acid (Fig. 1b). The carboxyl groups on both ends can coordinate to the open-metal sites thus anchoring the molecular gate. The central pyrene allows the molecule to rotate around an axle like a rotor. Finally, the negatively charged fluorine groups and the positively charged methyl groups create a permanent dipole which enables the molecule to respond changes in an electric field. Fig. 1c shows a molecular gate placed in one of the channels of Mg-MOF-74 and Fig. 1d shows all three channels of the MgMOF-74 occupied by the gate molecules.

\section{Methods}

\section{DFT calculations}

In order to assess the stability of a molecular gate mounted on Mg-MOF-74, we carried out DFT calculations. The crystal structure of Mg-MOF-74 was obtained from Cambridge Crystallographic Data Centre (CCDC) ${ }^{69}$ There are 162 atoms in a unit cell of Mg-MOF-74, and the unit cell dimensions and the angles are $a=25.873, b=25.873$, and $c=6.930$ and $\alpha=90.00$, $\beta=90.00$ and $\gamma=120.00$, respectively. For the DFT calculations, Mg-MOF-74 unit cell was replicated 2 times in the $z$-direction to give a $1 \times 1 \times 2$ structure. The gate was placed in one of the three channels of Mg-MOF-74 with the carboxyl groups positioned approximately $2.5 \AA$ away from the magnesium atoms (Fig. 1c). The MOF-molecular gate complex was then geometry optimized (flexible cell) with periodic planewave DFT calculations using ultrasoft pseudopotentials with the CASTEP 16.1 software ${ }^{70}$ with the gate molecule at different rotational angles with respect to the $x-y$ plane. Details of convergence criteria are given in Table S1. $\dagger$ The PBE functional ${ }^{71}$ with semi empirical dispersion corrections derived by Tkatchenko and Scheffler (DFT-D2) ${ }^{72}$ was used with the cut off energy and the $k$-point mesh set at $500 \mathrm{eV}$ and $1 \times 1 \times 1$, respectively. Binding energies of the gate molecule in the optimized configurations were calculated by subtracting the energies of the MOF and the gate from the energy of the MOF-molecular gate complex.

\section{MD simulations}

MD simulations were carried out with the GROMACS ${ }^{73}$ software in order to investigate the effect of an external electric field on the molecular gates mounted on Mg-MOF-74. 
Because of the external electric field an additional force is exerted on the atoms due to partial charges. This force is calculated according to the following formula,

$$
F=q E
$$

where $F$ is the force, $q$ is the partial atomic charges and $E$ is external electric field. The electric field was applied in the $z$ direction which Mg-MOF-74 channels run through. Mg-MOF74 unit cell was replicated 5 times in the $z$-direction to obtain a $1 \times 1 \times 5$ structure. We considered two different gated structures; in the first one a single gate molecule was placed in one of the channels (Fig. 1c); and in the second one, a gate molecule was placed in each channel, resulting in a structure with all three channels occupied by the gate molecules (Fig. 1d). Furthermore, for the fully gated complex we simulated a system with methane molecules present in order to investigate the possibility of controlling fluid transport in the channels of Mg-MOF-74. For this purpose a graphene wall was placed $2.5 \mathrm{~nm}$ away from the gate molecules, and the pore volume between the graphene wall and the molecular gates was saturated with methane such that the distance between the centre of methane molecules and the centre of atoms of MOF, graphene wall and the gate molecules was slightly larger than the sum of their van der Waals radii. In total 103 methane molecules were inserted. $\mathrm{UFF}^{74}$ force field was used to model the MOF and the gate molecules and the graphene wall. MOF and the gate molecules were treated as flexible bodies. Methane was modelled by using the TraPPE force field. ${ }^{75}$ The repeat ${ }^{76}$ method was used to derive partial atomic charges by fitting them against the periodic electrostatic potential of the optimized MOF-molecular gate complex at the closed configuration obtained from DFT calculations. Details of all force field parameters can be found in the ESI. $\dagger$ Duration of the MD simulations ranged from 1 to $30 \mathrm{~ns}$. Verlet velocity algorithm ${ }^{77}$ was employed to integrate Newton's equation of motion with a time step of $1 \mathrm{fs}$. The cut-off distance was set at $12 \AA$ A. MD simulations were run at $298 \mathrm{~K}$ and 0 bars in the NPT ensemble using a Nose-Hoover thermostat and barostat for the MOFmolecular gate systems with no methane molecules; whereas, the system with the graphene wall and the methane molecules were simulated in the NVT ensemble.

\section{Results and discussion}

Closed (Fig. 2a) and open (Fig. 2b) configurations of the MOFmolecular gate complex with one gate molecule were optimized with periodic dispersion corrected DFT calculations. Binding energies of the gate molecule in the closed and open configurations were calculated as $-357 \mathrm{~kJ} \mathrm{~mol}^{-1}$ and $-278 \mathrm{~kJ}$ $\mathrm{mol}^{-1}$, respectively. These indicate that both the closed and open gate configurations are stable and the gate molecule binds to the MOF strongly. Furthermore, the MOF-molecular gate complex preserved the original hexagonal shape of the $\mathrm{Mg}$ MOF-74 channels. For instance, for the closed gate configuration the cell dimensions and the angles showed small deviations with respect to the experimental values and changed from $a=25.873, b=25.873$, and $c=13.860$ to $a=26.526, b=26.345$ and $c=13.861 \AA$, and from $\alpha=90.00, \beta=90.00$ and $\gamma=120.00$ to $\alpha=90.30, \beta=89.68$ and $\gamma=121.16$, respectively.

Fig. 3 shows the changes in the total energy of the complex obtained by DFT calculations with respect to the closed configuration as the molecular gate rotates until it reaches to the open configuration. Two rotational barriers were identified, one is $20 \mathrm{~kJ} \mathrm{~mol}^{-1}$ and located around 40 degrees, and the other one is $73 \mathrm{~kJ} \mathrm{~mol}^{-1}$ and located at 75 degrees. Closed and open configurations of the MOF-molecular gate complex with onegate molecules were also optimized with classical MD simulations in the NPT ensemble. Binding energies of the molecular were $-203 \mathrm{~kJ} \mathrm{~mol}^{-1}$ and $-126 \mathrm{~kJ} \mathrm{~mol}^{-1}$ for the closed and open configurations, respectively. These values are lower than those obtained by the dispersion corrected DFT calculations; but still indicate the molecular gate is predicted to bind strongly to the MOF in classical MD simulations. The stability of the MOFmolecular gate complex with one gate molecule was further tested under increasing electric field strengths. It was found that the complex retained its structural integrity up to $8 \mathrm{~V} \mathrm{~nm}^{-1}$. On the other hand, the minimum electric field to overcome the intrinsic rotational barrier of a single molecular gate and bring it from the closed to open configuration was found to be $0.5 \mathrm{~V}$ $\mathrm{nm}^{-1}$. A system with all three channels occupied by molecular gates was also considered (Fig. 1d). Fig. 4 shows the total energy of this system with the electric field $\left(3 \mathrm{~V} \mathrm{~nm}^{-1}\right)$ turned on and off with consecutive periods of $1 \mathrm{~ns}$. The simulation started with molecular gates at their closed configurations and run for $1 \mathrm{~ns}$. a)

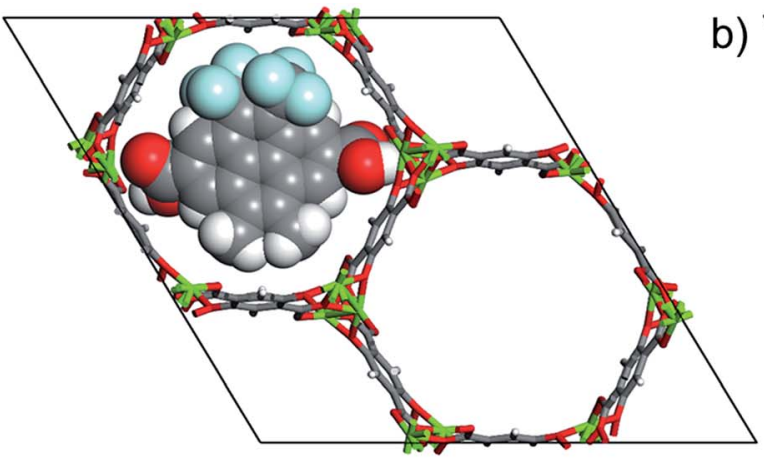

b)

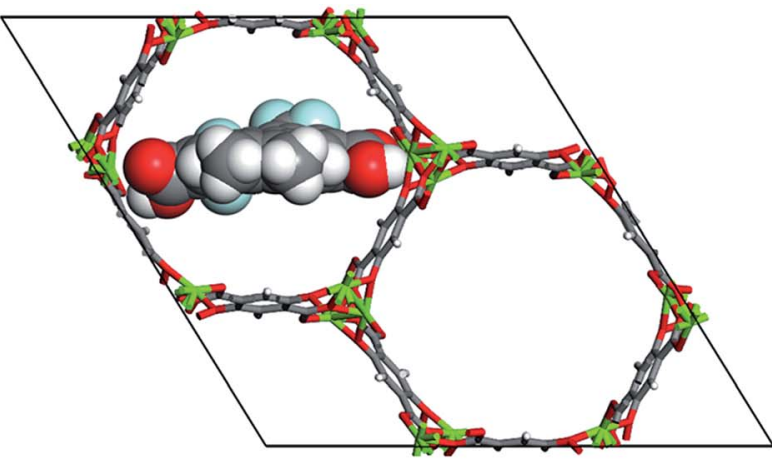

Fig. 2 (a) DFT optimized closed gate configuration and (b) open gate configuration. Atom colours are same with those in Fig. 1. 


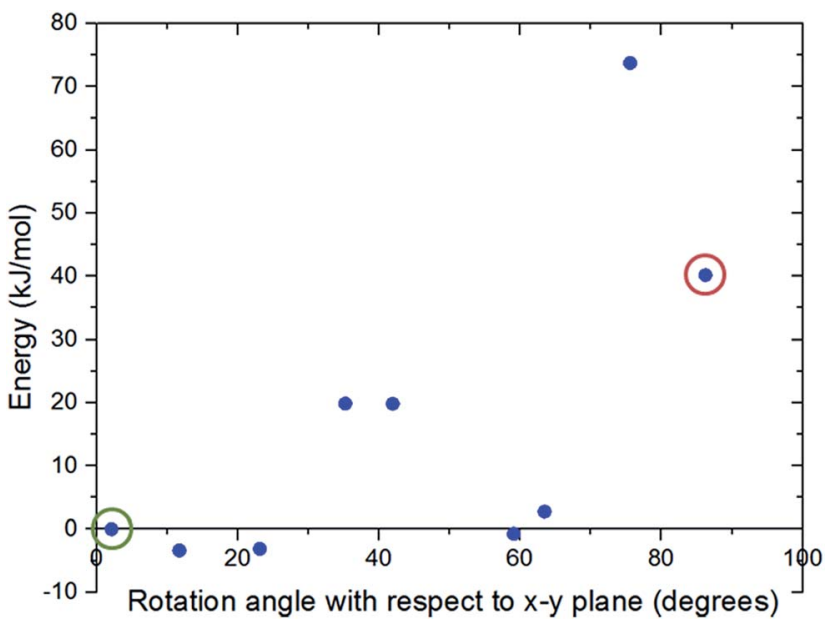

Fig. 3 Energy variation of the MOF-molecular gate complex with the rotation of the gate molecule with respect to the $x-y$ plane predicted by DFT calculations. The closed gate configuration, which was taken as the reference point, is circled with green and the open gate configuration with red.

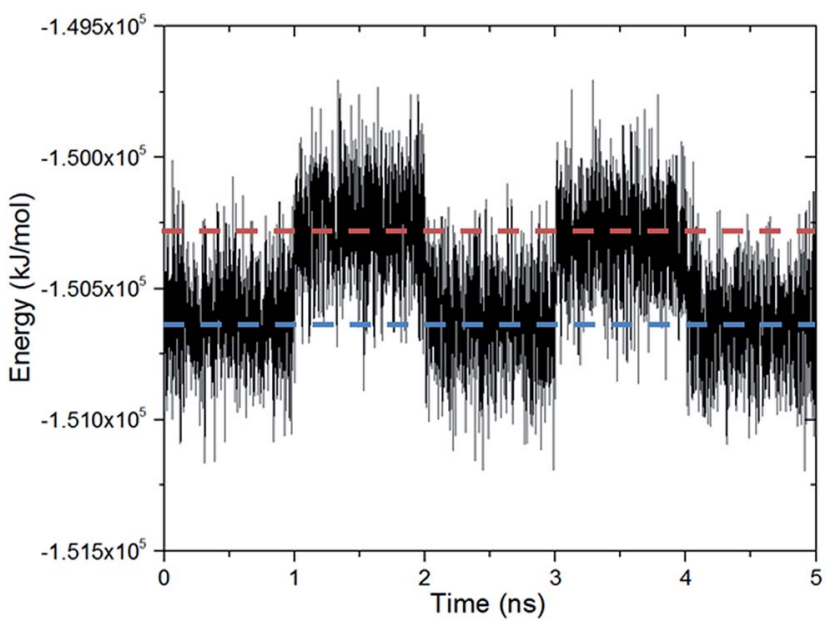

Fig. 4 The energy of the MOF-molecular gate complex with all three channels occupied by the molecular gates form the MD simulation. An electric field with a $3 \mathrm{~V} \mathrm{~nm}{ }^{-1}$ strength turned on and off with consecutive periods of $1 \mathrm{~ns}$. The gate is at the closed configuration when the electric field is turned off (blue line) and at the open configuration when the electric field is turned on (red line).

Then the electric field was turned on in the $z$-direction for $1 \mathrm{~ns}$ during which the molecular gates switched to their open configurations. When the electric field was turned off for the next $1 \mathrm{~ns}$, the molecular gates reverted back to their closed configurations. The energy difference between the closed and the open configurations was about $350 \mathrm{~kJ} \mathrm{~mol}^{-1}$.

The complex with all three channels occupied by the molecular gates was further tested with methane molecules placed between the gates and the graphene wall in an NVT ensemble MD simulation (Fig. 5a). First, the system was simulated with the gate molecules in the closed configuration for $30 \mathrm{~ns}$ and no electric field was applied. The molecular gates resisted to rotation despite the pressure due to the presence of methane molecules (Fig. S1 $\dagger$ )
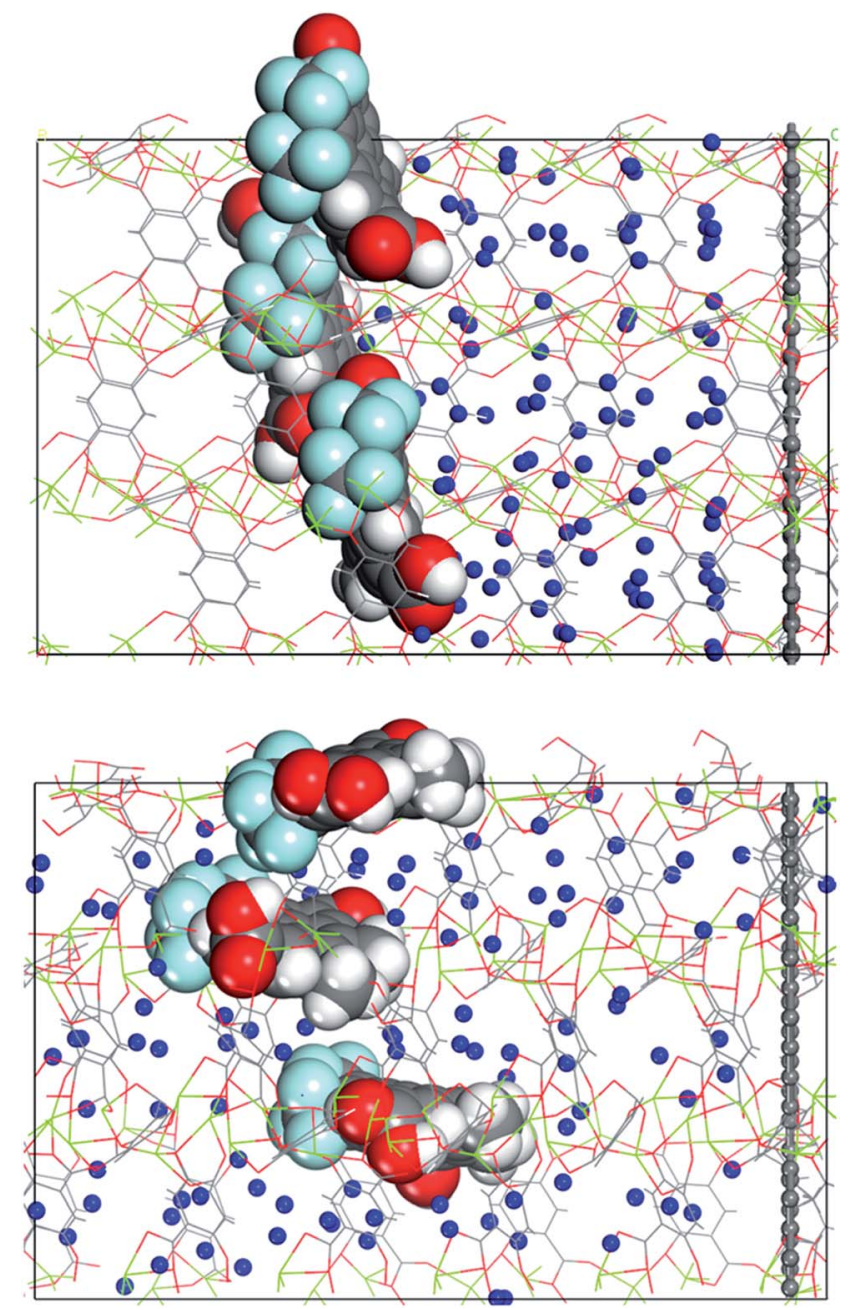

Fig. 5 (a) Methane molecules placed between the molecular gates and the graphene wall, and (b) molecular gates switched to their open configurations and the methane molecules diffused towards the empty pore volume after the electric field was turned on. Methane molecules (blue), all other atom colours are same with those in Fig. 1.

and remained in closed configuration as shown in Fig. 5a. During this period no methane molecules diffused to the empty pore volume behind the gates. This was visually confirmed and quantified with the number density of methane along the $z$ direction (Fig. 6a). Then the electric field in the $z$-direction was turned on $\left(3 \mathrm{~V} \mathrm{~nm}^{-1}\right)$ and the system was simulated for another 30 ns. With the electric field turned on, the molecular gates switched to open configuration (Fig. 5b) and the methane molecules diffused to the empty pore volume (Fig. 6b).

It should be noted that gate effects reported in the MOF literature are substantially different than the molecular gate concept we present in this study. Rather than involving a door type mechanism which opens and closes, previously reported gate effects were all based on either the reorientation of ligands or a shift in the topology of the structure, such that the pore volume decreased or increased up on exposure to external stimuli, e.g., gas adsorption or light. ${ }^{78-80}$ To the best of our knowledge, this is the first specific molecular gate design in MOFs dedicated to open and close the MOF pores for diffusion 

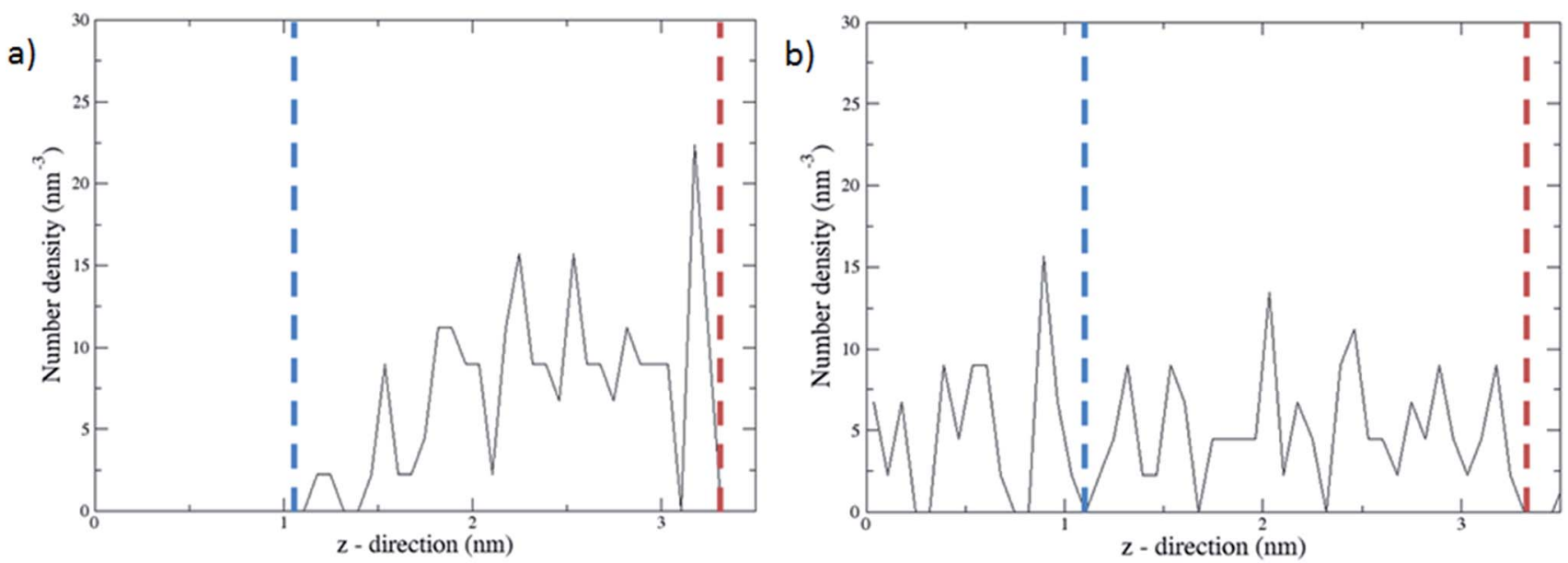

Fig. 6 Number densities of methane along the $z$-direction averaged over 30 ns with the molecular gates are in (a) closed configuration and (b) open configuration. The blue and red dashed lines represent the positions of the gate molecules and the graphene wall, respectively.

of molecules without the MOF undergoing any structural change. Overall, the MOF-molecular gate complex we designed resembles a butterfly valve mounted inside a pipe in order to regulate or prevent flow. By employing different functional groups it would be possible to adjust the electric field required to open or close this nanovalve. One could also imagine controlling the degree of opening of the valve by applying an electric field with different angles with respect to the $x-y$ plane and thus regulating the flow rate.

We recognise that the synthesis of a device which integrates a MOF and a molecular gate responding to an external electric field is a daunting task. However, there have been recent reports of experimental work which demonstrated the incorporation of molecular machines in MOFs. Stoddard and colleagues ${ }^{51,52}$ mounted rotaxane and catenane based molecular switches on the open-metal coordination sites of NU-1000 $\mathrm{MOF}^{81} \mathrm{Zhu}$ et al. synthesized a molecular shuttle in the form of a crown- 8 wheel which moved back and forth on the ligands of UWDM-4 MOF. ${ }^{82}$ The use of an electric field as an external stimulus has also been demonstrated. Zheng et al. ${ }^{21}$ used the tip of a scanning tunnelling microscope (STM) to create an electric field in order to switch the configuration of a fluorine functionalised phenanthrene molecular turnstile between two different stable positions. Remarkably, the strength of the electric field used in the study of Zheng et al., which was $1 \mathrm{~V} \mathrm{~nm}^{-1}$, compares very well with the range of electric field we used in our computational study. In another ground breaking study, again an STM tip was used to drag a molecular car over a distance of several nanometres. ${ }^{18}$ At the very least these experimental studies suggest that the incorporation of more complex molecular machines controlled by an external electric field in MOFs, or in other porous materials, such as the molecular gate we propose, can one day go beyond the level of conceptual design based on molecular simulations and become reality.

\section{Conclusions}

By taking advantage of open-metal coordination sites in MOFs we computationally designed a molecular gate mounted on
MOFs. Mg-MOF-74, a MOF with hexagonal channels with openmetal coordination sites at each corner, was used as the host material. For the gate molecule 4,5-dimethyl-9,10bis(trifloromethyl)pyrene-2,7-dicarboxylic acid was considered. This gate molecule has carboxyl groups to coordinate to the open-metal sites, a permanent dipole to respond to an electric field and a pyrene backbone to act as an axle. By carrying out dispersion corrected DFT calculations we showed that the gate molecule binds to the MOF strongly. MD simulations demonstrated that the gate molecules switch between two stable configurations, closed and open, by turning on and off an electric field. We then showed that this can be used to control the flow of methane molecules in the channels of Mg-MOF-74. The concept of electric field controlled molecular gates mounted on MOFs presented in this study can inspire the rational design of new molecular machines and nanodevices which can store, deliver and select molecules on demand and with atomic precision.

\section{Acknowledgements}

B. J. acknowledges Engineering and Physical Sciences Research Council (EPSRC) for supporting graduate studies. O. Y. acknowledges financial support from Leverhulme Trust Grant RPG-2016-331.

\section{References}

1 W. R. Browne and B. L. Feringa, Nat. Nanotechnol., 2006, 1, 25-35.

2 J. Cao, M. C. T. Fyfe, J. F. Stoddart, G. R. L. Cousins and P. T. Glink, J. Org. Chem., 2000, 65, 1937-1946.

3 J. Conyard, K. Addison, I. A. Heisler, A. Cnossen, W. R. Browne, B. L. Feringa and S. R. Meech, Nat. Chem., 2012, 4, 547-551.

4 C. O. Dietrich-Buchecker, J. P. Sauvage and J. P. Kintzinger, Tetrahedron Lett., 1983, 24, 5095-5098. 
5 P. L. Anelli, N. Spencer and J. F. Stoddart, J. Am. Chem. Soc., 1991, 113, 5131-5133.

6 N. Koumura, R. W. J. Zijlstra, R. A. van Delden, N. Harada and B. L. Feringa, Nature, 1999, 401, 152-155.

7 J. Seo, R. Matsuda, H. Sakamoto, C. Bonneau and S. Kitagawa, J. Am. Chem. Soc., 2009, 131, 12792-12800.

8 S. Muramatsu, K. Kinbara, H. Taguchi, N. Ishii and T. Aida, J. Am. Chem. Soc., 2006, 128, 3764-3769.

9 J. D. Badjić, V. Balzani, A. Credi, S. Silvi and J. F. Stoddart, Science, 2004, 303, 1845.

10 J. D. Badjic, C. M. Ronconi, J. F. Stoddart, V. Balzani, S. Silvi and A. Credi, J. Am. Chem. Soc., 2006, 128, 1489-1499.

11 B. J. Dahl and B. P. Branchaud, Tetrahedron Lett., 2004, 45, 9599-9602.

12 R. A. van Delden, M. K. J. Ter Wiel, M. M. Pollard, J. Vicario, N. Koumura and B. L. Feringa, Nature, 2005, 437, 1337-1340.

13 N. Ruangsupapichat, M. M. Pollard, S. R. Harutyunyan and B. L. Feringa, Nat. Chem., 2011, 3, 53-60.

14 M. Lindqvist, K. Borre, K. Axenov, B. Kótai, M. Nieger, M. Leskelä, I. Pápai and T. Repo, J. Am. Chem. Soc., 2015, 137, 4038-4041.

15 S. Erbas-Cakmak, D. A. Leigh, C. T. McTernan and A. L. Nussbaumer, Chem. Rev., 2015, 115, 10081-10206.

16 E. R. Kay, D. A. Leigh and F. Zerbetto, Angew. Chem., Int. Ed., 2007, 46, 72-191.

17 R. Eelkema, M. M. Pollard, J. Vicario, N. Katsonis, B. S. Ramon, C. W. M. Bastiaansen, D. J. Broer and B. L. Feringa, Nature, 2006, 440, 163.

18 T. Kudernac, N. Ruangsupapichat, M. Parschau, B. Macia, N. Katsonis, S. R. Harutyunyan, K.-H. Ernst and B. L. Feringa, Nature, 2011, 479, 208-211.

19 A. V. Akimov and A. B. Kolomeisky, J. Phys. Chem. C, 2012, 116, 22595-22601.

20 T. C. Bedard and J. S. Moore, J. Am. Chem. Soc., 1995, 117, 10662-10671.

21 X. Zheng, M. E. Mulcahy, D. Horinek, F. Galeotti, T. F. Magnera and J. Michl, J. Am. Chem. Soc., 2004, 126, 4540-4542.

22 D. Horinek and J. Michl, Proc. Natl. Acad. Sci. U. S. A., 2005, 102, 14175-14180.

23 L.-Y. Hsu, E. Y. Li and H. Rabitz, Nano Lett., 2013, 13, 50205025.

24 J. S. Seldenthuis, F. Prins, J. M. Thijssen and H. S. J. van der Zant, ACS Nano, 2010, 4, 6681-6686.

25 H. Li, M. Eddaoudi, M. O'Keeffe and O. M. Yaghi, Nature, 1999, 402, 276-279.

26 H.-C. Zhou and S. Kitagawa, Chem. Soc. Rev., 2014, 43, 54155418.

27 J. R. Long and O. M. Yaghi, Chem. Soc. Rev., 2009, 38, 12131214.

28 R. J. Kuppler, D. J. Timmons, Q.-R. Fang, J.-R. Li, T. A. Makal, M. D. Young, D. Yuan, D. Zhao, W. Zhuang and H.-C. Zhou, Coord. Chem. Rev., 2009, 253, 3042-3066.

29 H. Furukawa, K. E. Cordova, M. O'Keeffe and O. M. Yaghi, Science, 2013, 341, 1230444.

30 S. Qiu, M. Xue and G. Zhu, Chem. Soc. Rev., 2014, 43, 61166140 .
31 L. Li, S. Tang, C. Wang, X. Lv, M. Jiang, H. Wu and X. Zhao, Chem. Commun., 2014, 50, 2304-2307.

32 D. Alezi, Y. Belmabkhout, M. Suyetin, P. M. Bhatt, Ł. J. Weseliński, V. Solovyeva, K. Adil, I. Spanopoulos, P. N. Trikalitis, A.-H. Emwas and M. Eddaoudi, J. Am. Chem. Soc., 2015, 137, 13308-13318.

33 J. Zhuang, C.-H. Kuo, L.-Y. Chou, D.-Y. Liu, E. Weerapana and C.-K. Tsung, ACS Nano, 2014, 8, 2812-2819.

34 T. Kundu, S. Mitra, P. Patra, A. Goswami, D. Díaz Díaz and R. Banerjee, Chem.-Eur. J., 2014, 20, 10514-10518.

35 D. Ma, B. Li, X. Zhou, Q. Zhou, K. Liu, G. Zeng, G. Li, Z. Shi and S. Feng, Chem. Commun., 2013, 49, 8964-8966.

36 J.-H. Wang, M. Li and D. Li, Chem. Sci., 2013, 4, 1793-1801. 37 M.-J. Dong, M. Zhao, S. Ou, C. Zou and C.-D. Wu, Angew. Chem., Int. Ed., 2014, 53, 1575-1579.

38 A. Herbst, A. Khutia and C. Janiak, Inorg. Chem., 2014, 53, 7319-7333.

39 P. Garcia-Garcia, M. Muller and A. Corma, Chem. Sci., 2014, 5, 2979-3007.

40 A. Dhakshinamoorthy and H. Garcia, Chem. Soc. Rev., 2014, 43, 5750-5765.

41 Z. Wang and S. M. Cohen, Angew. Chem., 2008, 120, 47774780 .

42 Z. Wang and S. M. Cohen, Chem. Soc. Rev., 2009, 38, 13151329.

43 B. Li, Y. Zhang, D. Ma, L. Li, G. Li, G. Li, Z. Shi and S. Feng, Chem. Commun., 2012, 48, 6151-6153.

44 T. M. McDonald, D. M. D'Alessandro, R. Krishna and J. R. Long, Chem. Sci., 2011, 2, 2022-2028.

45 A. Ö. Yazaydın, A. I. Benin, S. A. Faheem, P. Jakubczak, J. J. Low, R. R. Willis and R. Q. Snurr, Chem. Mater., 2009, 21, 1425-1430.

46 S.-T. Zheng, X. Zhao, S. Lau, A. Fuhr, P. Feng and X. Bu, J. Am. Chem. Soc., 2013, 135, 10270-10273.

47 Y.-Y. Fu, C.-X. Yang and X.-P. Yan, Langmuir, 2012, 28, 67946802.

48 Y. K. Hwang, D.-Y. Hong, J.-S. Chang, S. H. Jhung, Y.-K. Seo, J. Kim, A. Vimont, M. Daturi, C. Serre and G. Férey, Angew. Chem., Int. Ed., 2008, 47, 4144-4148.

49 A. Torrisi, R. G. Bell and C. Mellot-Draznieks, Cryst. Growth Des., 2010, 10, 2839-2841.

50 C. Zlotea, D. Phanon, M. Mazaj, D. Heurtaux, V. Guillerm, C. Serre, P. Horcajada, T. Devic, E. Magnier, F. Cuevas, G. Ferey, P. L. Llewellyn and M. Latroche, Dalton Trans., 2011, 40, 4879-4881.

51 P. R. McGonigal, P. Deria, I. Hod, P. Z. Moghadam, A.-J. Avestro, N. E. Horwitz, I. C. Gibbs-Hall, A. K. Blackburn, D. Chen, Y. Y. Botros, M. R. Wasielewski, R. Q. Snurr, J. T. Hupp, O. K. Farha and J. F. Stoddart, Proc. Natl. Acad. Sci. U. S. A., 2015, 112, 11161-11168.

52 Q. Chen, J. Sun, P. Li, I. Hod, P. Z. Moghadam, Z. S. Kean, R. Q. Snurr, J. T. Hupp, O. K. Farha and J. F. Stoddart, J. Am. Chem. Soc., 2016, 138, 14242-14245.

53 G. P. Moss, Pure Appl. Chem., 1998, 70, 143.

54 E. D. Bloch, W. L. Queen, R. Krishna, J. M. Zadrozny, C. M. Brown and J. R. Long, Science, 2012, 335, 1606-1610. 
55 T. Grant Glover, G. W. Peterson, B. J. Schindler, D. Britt and O. Yaghi, Chem. Eng. Sci., 2011, 66, 163-170.

56 W. Zhou, H. Wu and T. Yildirim, J. Am. Chem. Soc., 2008, 130, 15268-15269.

57 Z. Bao, L. Yu, Q. Ren, X. Lu and S. Deng, J. Colloid Interface Sci., 2011, 353, 549-556.

58 H. Wu, W. Zhou and T. Yildirim, J. Am. Chem. Soc., 2009, 131, 4995-5000.

59 T. Pham, K. A. Forrest, J. Eckert and B. Space, Cryst. Growth Des., 2016, 16, 867-874.

60 T. Pham, K. A. Forrest, K. McLaughlin, J. Eckert and B. Space, J. Phys. Chem. C, 2014, 118, 22683-22690.

61 A. L. Dzubak, L.-C. Lin, J. Kim, J. A. Swisher, R. Poloni, S. N. Maximoff, B. Smit and L. Gagliardi, Nat. Chem., 2012, 4, 810-816.

62 J. Liu, A. I. Benin, A. M. B. Furtado, P. Jakubczak, R. R. Willis and M. D. LeVan, Langmuir, 2011, 27, 11451-11456.

63 P. Verma, X. Xu and D. G. Truhlar, J. Phys. Chem. C, 2013, 117, 12648-12660.

64 H. Kim, J. Park and Y. Jung, Phys. Chem. Chem. Phys., 2013, 15, 19644-19650.

65 S. M. Chavan, G. C. Shearer, E. Bloch and S. Bordiga, ChemPhysChem, 2012, 13, 445-448.

66 D. Yu, A. O. Yazaydin, J. R. Lane, P. D. C. Dietzel and R. Q. Snurr, Chem. Sci., 2013, 4, 3544-3556.

67 K. Yu, K. Kiesling and J. R. Schmidt, J. Phys. Chem. C, 2012, 116, 20480-20488.

68 P. Canepa, C. A. Arter, E. M. Conwill, D. H. Johnson, B. A. Shoemaker, K. Z. Soliman and T. Thonhauser, J. Mater. Chem. A, 2013, 1, 13597-13604.

69 W. L. Queen, M. R. Hudson, E. D. Bloch, J. A. Mason, M. I. Gonzalez, J. S. Lee, D. Gygi, J. D. Howe, K. Lee, T. A. Darwish, M. James, V. K. Peterson, S. J. Teat, B. Smit,
J. B. Neaton, J. R. Long and C. M. Brown, Chem. Sci., 2014, $5,4569-4581$.

70 J. Clark Stewart, D. Segall Matthew, J. Pickard Chris, J. Hasnip Phil, I. J. Probert Matt, K. Refson and C. Payne Mike, Z. Kristallogr., 2005, 220, 567.

71 J. P. Perdew, K. Burke and M. Ernzerhof, Phys. Rev. Lett., 1996, 77, 3865-3868.

72 A. Tkatchenko and M. Scheffler, Phys. Rev. Lett., 2009, 102, 073005.

73 M. J. Abraham, T. Murtola, R. Schulz, S. Páll, J. C. Smith, B. Hess and E. Lindahl, SoftwareX, 2015, 1-2, 19-25.

74 C. J. Casewit, K. S. Colwell and A. K. Rappe, J. Am. Chem. Soc., 1992, 114, 10035-10046.

75 J. J. Potoff and J. I. Siepmann, AIChE J., 2001, 47, 1676-1682. 76 C. Campañá, B. Mussard and T. K. Woo, J. Chem. Theory Comput., 2009, 5, 2866-2878.

77 W. C. Swope, H. C. Andersen, P. H. Berens and K. R. Wilson, J. Chem. Phys., 1982, 76, 637-649.

78 D. Tanaka, K. Nakagawa, M. Higuchi, S. Horike, Y. Kubota, T. C. Kobayashi, M. Takata and S. Kitagawa, Angew. Chem., 2008, 120, 3978-3982.

79 R. Lyndon, K. Konstas, A. W. Thornton, A. J. Seeber, B. P. Ladewig and M. R. Hill, Chem. Mater., 2015, 27, 78827888.

80 D. Fairen-Jimenez, S. A. Moggach, M. T. Wharmby, P. A. Wright, S. Parsons and T. Düren, J. Am. Chem. Soc., 2011, 133, 8900-8902.

81 J. E. Mondloch, W. Bury, D. Fairen-Jimenez, S. Kwon, E. J. DeMarco, M. H. Weston, A. A. Sarjeant, S. T. Nguyen, P. C. Stair, R. Q. Snurr, O. K. Farha and J. T. Hupp, J. Am. Chem. Soc., 2013, 135, 10294-10297.

82 K. Zhu, C. A. O'Keefe, V. N. Vukotic, R. W. Schurko and S. J. Loeb, Nat. Chem., 2015, 7, 514-519. 